\title{
Giant non-functional parathyroid cyst: A case report
}

\author{
XU ZHANG ${ }^{1}$, JUN-HUI YUAN ${ }^{2}$, LU FENG $^{1}$, DONG-QIU SHAN ${ }^{2}$, JUN-FU WU ${ }^{1}$ and SHAN-TING LIU ${ }^{1}$ \\ Departments of ${ }^{1}$ Head, Neck and Thyroid, and ${ }^{2}$ Radiology, Affiliated Tumor Hospital, \\ Zhengzhou University, Zhengzhou, Henan 450008, P.R. China
}

Received March 15, 2015; Accepted December 23, 2015

DOI: $10.3892 / \mathrm{ol} .2016 .4181$

\begin{abstract}
Giant parathyroid cysts (PCs) are a rare entity and possess a benign clinical course. PCs may be functional or non-functional, depending on the ability of the cyst to secrete parathyroid hormone (PTH). The present study reports a rare case of a giant PC in a 56-year-old male who presented to the Affiliated Tumor Hospital, Zhengzhou University (Zhengzhou, Henan, China) with a 10-month history of exertional dyspnea, associated with mild dysphagia that had persisted for 3 months. The present study reviews the clinical situation, laboratory examination, radiographic findings, treatment and prognosis of the patient, and provides a brief discussion regarding the associated literature. Giant PCs may manifest with compressive symptoms of the surrounding tissues. The diagnosis of a giant PC is based on increased levels of PTH in the fluid collected during the aspiration of the cyst. Management by surgical excision is recommended for giant PCs that cause local cervical symptoms.
\end{abstract}

\section{Introduction}

Parathyroid cysts (PCs) were first reported in 1880 in a study by Sandstrom, but PC removal from the neck was first reported in 1905 in a study by Goris $(1,2)$. PCs account for $0.6 \%$ of all thyroid and parathyroid masses. These lesions always arise in the posterior neck or anterior mediastinum $(3,4)$. PCs may be functional or non-functional, depending on the ability of the cyst to secrete parathyroid hormone (PTH). In total, $15-57 \%$ of PCs are functional (5-7). In addition, the majority of non-functional PC lesions are small in size and are occasionally revealed during imaging procedures or cervical surgery, with only a minority characterized by a large size and compressive symptoms (8). The present study describes the case of a man with a giant non-functional PC extending from the lower neck

Correspondence to: Professor Shan-Ting Liu, Department of Head, Neck and Thyroid, Affiliated Tumor Hospital, Zhengzhou University, 127 Dongming Road, Zhengzhou, Henan 450008, P.R. China

E-mail: hnszlyyzx@126.com

Key words: parathyroid cyst, non-functioning, parathyroid lesion, mediastinal lesion, surgical excision to the superior mediastinum, and provides a brief discussion regarding the associated literature in order to raise awareness of this diagnosis.

\section{Case report}

A 56-year-old man was referred to the Affiliated Tumor Hospital, Zhengzhou University (Zhengzhou, Henan, China) in September 2014 with a 10 month history of exertional dyspnea, and mild dysphagia that had persisted for 3 months. The medical history of the patient included hypertension and an inguinal hernia repair, which had occurred 10 years previously.

The physical examination revealed a palpable round-shaped mass in the right thyroid lobe. The ultrasound (Philips iU22; Philips Healthcare, Andover, MA, USA) showed a $8.4 \times 5.7 \times 3.6-\mathrm{cm}$ cystic mass attached to the right thyroid lobe (Fig. 1). A neck and chest computed tomography (CT; LightSpeed Pro32 spiral scanner with a layer thickness of $5 \mathrm{~mm}$; GE Healthcare Bio-Sciences, Pittsburgh, PA, USA) scan was performed with contrast media, which showed the presence of a large neck and mediastinal mass $(9.0 \times 6.0 \mathrm{~cm})$ with no contrast enhancement, causing right anterolateral displacement of the large vessels and left anterolateral displacement of the trachea (Fig. 2). The serum calcium and PTH concentrations were measured and identified as normal, at $2.26 \mathrm{mmol} / \mathrm{l}$ (normal range, 2.03-2.54 $\mathrm{mmol} / \mathrm{l}$ ) and $37.98 \mathrm{pg} / \mathrm{ml}$ (normal range, $15-65 \mathrm{pg} / \mathrm{m})$, respectively. A fine-needle aspiration biopsy (FNAB) was performed, and the content obtained from the cyst was a colorless crystal clear fluid, suggesting that the mass was a PC. The levels of PTH in the aspirated fluid were elevated (308 pg/ml; normal serum range, 10-65 pg/ml). A cytological examination of the material that was obtained following centrifugation of the fluid showed a few epithelial cells that possibly belonged to the parathyroid tissue. Based on the aforementioned findings, the diagnosis of the giant cyst was of a non-functional PC.

The patient underwent a right thyroid lobectomy and isthmectomy, with the excision of the cyst. The appearance of the giant PC was characterized by large cystic lesions with a thin wall and a watery fluid component (Fig. 3).

The cystic wall tissue was fixed in $10 \%$ formalin, washed and dehydrated using alcohol and xylene, paraffin-embedded, and stained with hematoxylin and eosin (Zhuhai Beisuo Biological Technology Co., Ltd., Zhuhai, China) for examination. The histological examination confirmed the presence of 


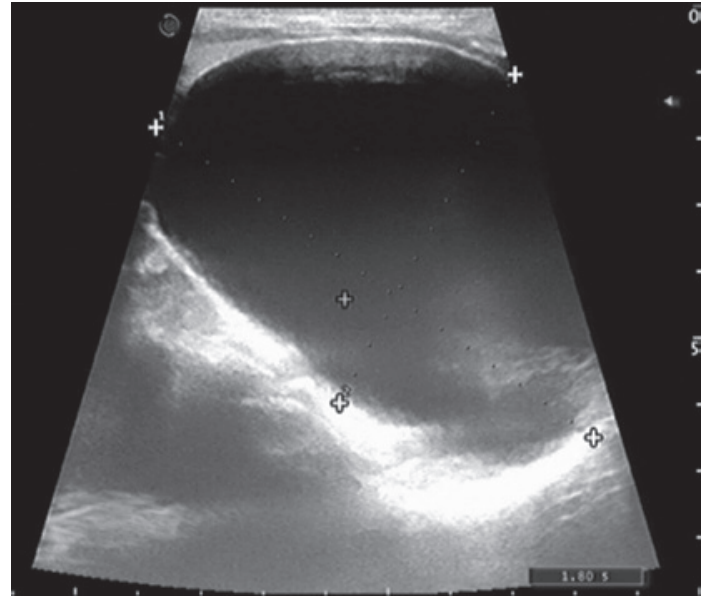

Figure 1. Sagittal view of the thyroid gland on ultrasound, showing the presence of a $80-\mathrm{mm}$ cystic nodule in the right lobe.

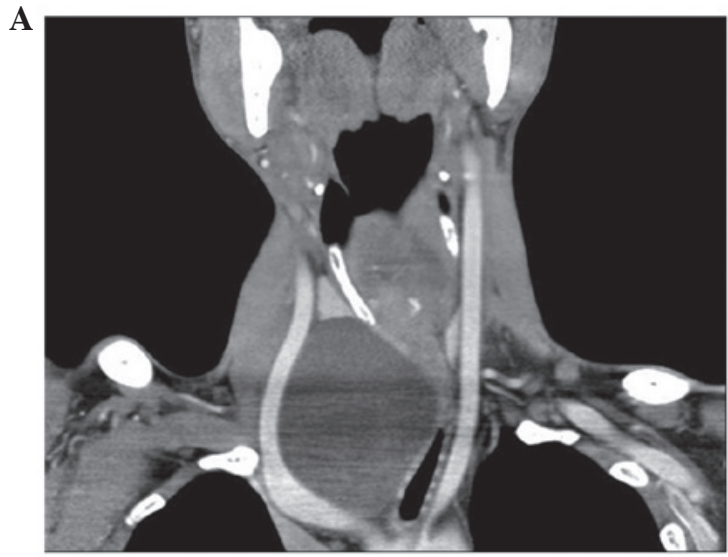

B

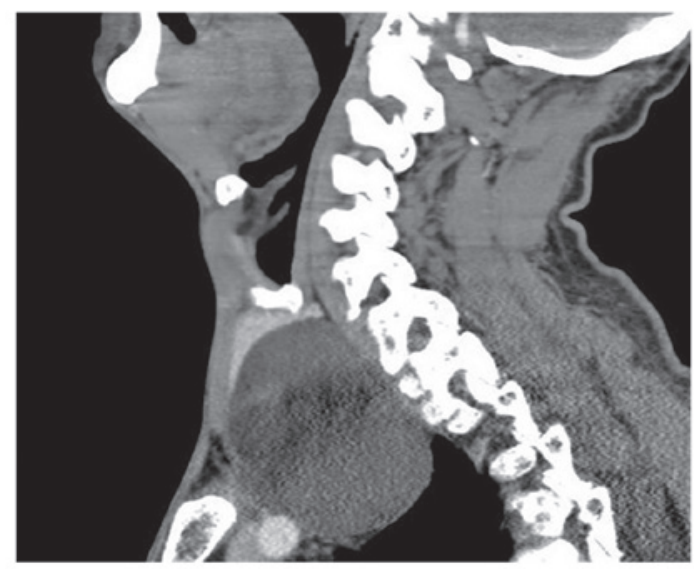

Figure 2. Neck and chest computed tomography scans with contrast media revealing a large mediastinal mass $(9 \times 6 \mathrm{~cm})$ without contrast enhancement causing the right anterolateral displacement of large vessels and the left anterolateral displacement of the trachea. (A) Coronal scanning. (B) Sagittal scanning.

a giant PC. The cyst was surrounded by a fibrous wall and possessed the focal presence of hyperplastic parathyroid cells within the fibrotic tissue. Neither mitotic figures nor nuclear atypia were observed (Fig. 4).

No serious complications occurred subsequent to the surgery. The calcium and PTH levels of the patient were monitored post-operatively, and no abnormalities were identified.
A

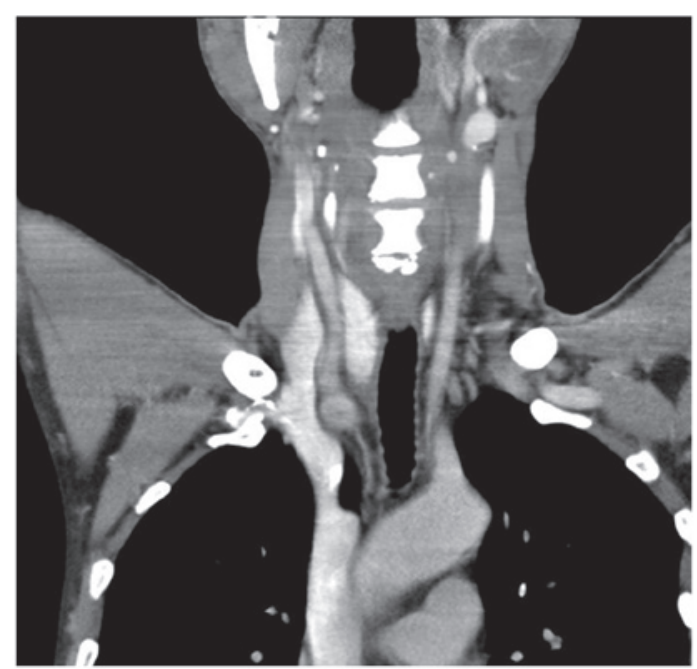

B

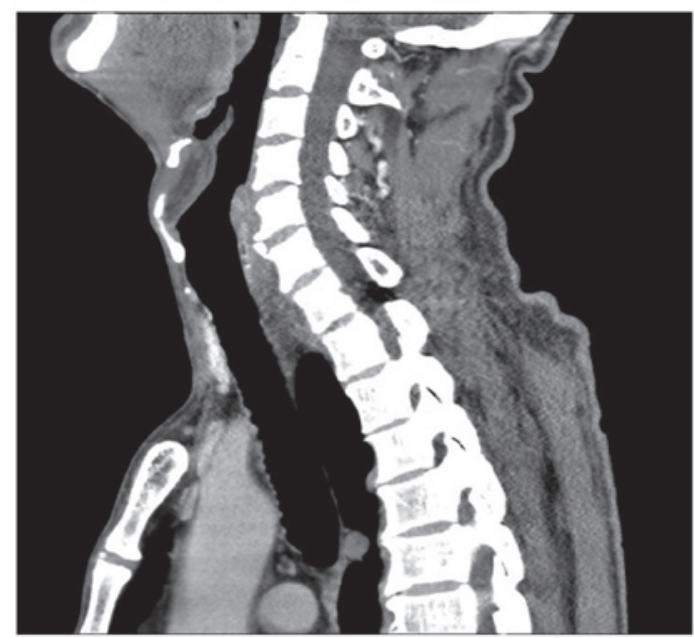

Figure 3. Post-operative computed tomography scan showing that the giant parathyroid cyst had been removed, and that the displaced trachea and large vessels had returned to the normal positions. (A) Coronal scanning. (B) Sagittal scanning.

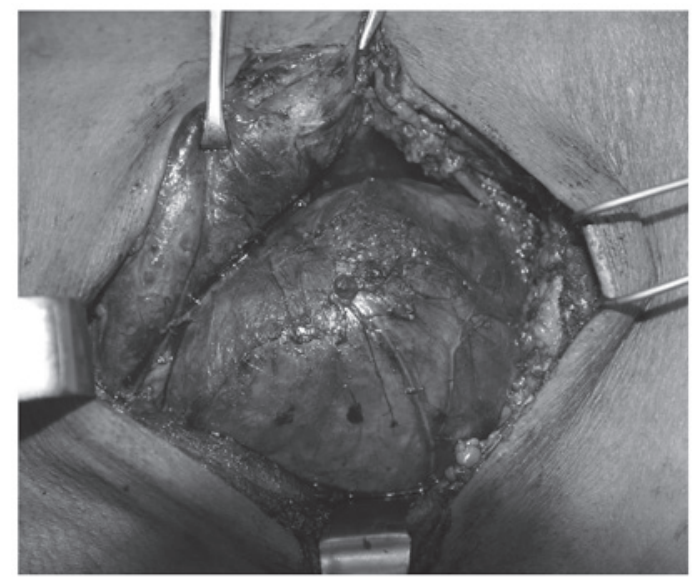

Figure 4. Gross appearance of the cystic parathyroid lesion.

Symptoms caused by the cyst were relieved immediately following surgery. The patient was discharged 7 days after the surgery (Fig. 5). The patient remains asymptomatic, with no recurrence at 3-month follow-up. 


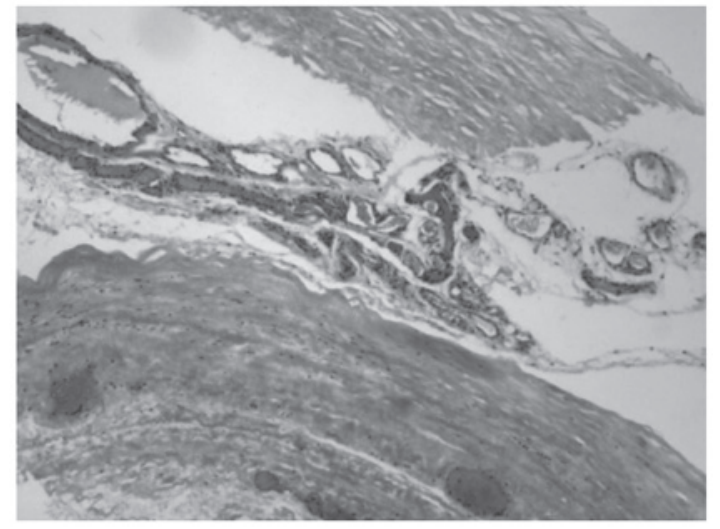

Figure 5. Histological features of a non-functioning parathyroid cyst, including a fibrous cystic wall with adjacent nests of clear or chief cells. Hematoxylin and eosin staining; magnification, $\mathrm{x} 250$.

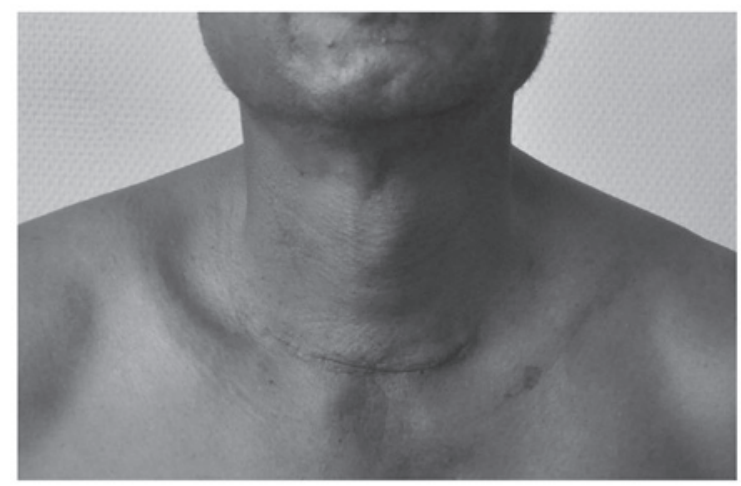

Figure 6. Appearance of the well-healed wounds at 7 days post-surgery.

\section{Discussion}

PCs are rare, having been reported in just over 300 cases and possessing an incidence ranging between $0.075-3.000 \%$ worldwide, as stated by previous studies $(9,10)$. Giant PCs that extend between the lower neck and superior mediastinum are even rarer. McKay et al (11) reported that in all of the PubMed studies that were published since 1925, only 107 giant non-functioning PCs had been reported. The study regarding large parathyroid cystic lesions that was conducted by Rossi et al (12) reported a $0.09 \%$ incidence over the last 15 years.

PCs may be misinterpreted as thyroid cystic lesions due to the close spatial association with the thyroid gland. Current diagnostic tools, including US, technetium-99m sestamibi scanning, CT and magnetic resonance imaging, may often differentiate between solid tumors and cystic lesions; however, differentiating between thyroid cysts and PCs is challenging. FNAB is a valuable diagnostic tool for the morphological evaluation of solid or cystic thyroid and parathyroid lesions (13). The typical findings of a watery, colorless crystal clear fluid are, in the majority of cases, suggestive of the diagnosis of a non-functional PC. The diagnosis may be established by elevated levels of PTH in the aspirated fluid (14).

Clinically, no symptoms are usually associated with PCs that are located in the parathyroid region and are not excessively large or causing compression of the adjacent tissue. Therefore, PCs tend to be large at the initial identification. In the present study, the cyst caused dyspnea in the patient as a result of marked tracheal deviation, and dysphagia due to esophageal deviation.

Controversy exists regarding the treatment of PCs. Until the 1970s, surgical treatment was considered to be the optimal scheme (15). Since the 1970s, certain studies have indicated that FNAB is the preferred management scheme due to the rarity of recurrence of PCs subsequent to aspiration (16). By contrast, a study by Sung et al stated that only $33 \%$ of non-functioning lesions were successfully treated by a simple aspiration alone (17). Similarly, Ujiki et al reported that the majority of cysts recurred subsequent to aspiration, and that surgery may be a more beneficial treatment (18). However, to the best of our knowledge, the treatment of non-functioning PCs may be considered in association with the size of the cyst. In the present study, the giant PC caused compressive symptoms; therefore, managing the $\mathrm{PC}$ with surgical therapy was essential.

For PCs that extend to the mediastinum, common surgical strategies include video-assisted thoracic surgery (VATS), a standard posterolateral thoracotomy and the classical cervical anterior approach. The VATS and posterolateral thoracotomy surgical strategies were excluded for the present study due to the size of the cyst and the surgical trauma that would be caused. In our experience, the classical cervical anterior approach should be used on PCs that are $<10 \mathrm{~cm}$ in diameter. In order to reduce the chance of relapse, the rupture of PCs should be prevented from occurring intraoperatively. If necessary, a region of the sternocleidomastoid muscle may be temporarily severed to avoid rupture.

To the best of our knowledge, no associated fatalities of treated patients have been documented in the previous literature and the resection of giant PCs has rarely been associated with post-operative complications (19).

In conclusion, the present study reported the case of a man with a giant non-functional PC extending from the lower neck to the superior mediastinum. Giant PCs are uncommon entities in clinical practice that may manifest with compressive symptoms of the surrounding tissues. The vast majority of PCs are non-functioning. The diagnosis of a PC may be confirmed by the detection of increased levels of PTH in the aspirated fluid, which in the majority of non-functional PCs is a watery, colorless crystal clear fluid. Surgery is the treatment of choice for giant non-functioning PCs, and the classical cervical anterior approach is recommended for use on PCs that are $<10 \mathrm{~cm}$ in diameter.

\section{References}

1. Sandstrom I: Whether humans have multiple thyroid lobes? Ups Lakafor Forhandl 14: 441-471, 1880 (In Danish).

2. Goris D: Excise the third thyroid lobe. Ann Soc Belg Chir 5: 394-400, 1905 (In French).

3. Mostafapour SP, True L and Futran ND: Clinical problem solving: Pathology. Pathology quiz case 1: Benign parathyroid cyst. Arch Otolaryngol Head Neck Surg 128: 592-594, 594, 2002.

4. Pinney SP and Daly PA: Parathyroid cyst: An uncommon cause of a palpable neck mass and hypercalcemia. West J Med 170: 118-120, 1999.

5. Clark OH: Parathyroid cysts. Am J Surg 135: 395-402, 1978. 
6. Rosenberg J, Orlando R III,Ludwig M and Pyrtek LJ: Parathyroid cysts. Am J Surg 143: 473-480, 1982.

7. Ramos-Gabatin A, Young RL and Schenk D: Parathyroid cyst: Medical diagnosis and therapy. South Med J 75: 1138-1140, 1982.

8. Sen P, Flower N, Papesch M, Davis A and Spedding AV: A benign parathyroid cyst presenting with hoarse voice. J Laryngol Otol 114: 147-148, 2000.

9. McCoy KL, Yim JH, Zuckerbraun BS, Ogilvie JB, Peel RL and Carty SE: Cystic parathyroid lesions: Functional and nonfunctional parathyroid cysts. Arch Surg 144: 52-56, discussion 56, 2009.

10. Cappelli C, Rotondi M, Pirola I, De Martino E, Leporati P, Magri F, Rosei EA, Chiovato L and Castellano M: Prevalence of parathyroid cysts by neck ultrasound scan in unselected patients. J Endocrinol Invest 32: 357-359, 2009.

11. McKay GD, Ng TH, Morgan GJ and Chen RC: Giant functioning parathyroid cyst presenting as a retrosternal goitre. ANZ J Surg 77: 297-304, 2007.

12. Rossi ED, Revelli L, Giustozzi E, Straccia P, Stigliano E, Lombardi CP, Pontecorvi A and Fadda G: Large non-functioning parathyroid cysts: Our institutional experience of a rare entity and a possible pitfall in thyroid cytology. Cytopathology 26: 114-121, 2015.
13. Fadda G and Rossi ED: Liquid-based cytology in fine-needle aspiration biopsies of the thyroid gland. Acta Cytol 55: 389-400, 2011.

14. Ihm PS, Dray T, Sofferman RA, Nathan M and Hardin NJ: Parathyroid cysts: Diagnosis and management. Laryngoscope 111: 1576-1578, 2001.

15. Pacini F, Antonelli A, Lari R, Gasperini L, Baschieri L and Pinchera A: Unsuspected parathyroid cysts diagnosed by measurement of thyroglobulin and parathyroid hormone concentrations in fluid aspirates. Ann Intern Med 102: 793-794, 1985.

16. Shi B, Guo H and Tang N: Treatment of parathyroid cysts with fine-needle aspiration. Ann Intern Med 131: 797-798, 1999.

17. Sung JY, Baek JH, Kim KS, Lee D, Ha EJ and Lee JH: Symptomatic nonfunctioning parathyroid cysts: Role of simple aspiration and ethanol ablation. Eur J Radiol 82: 316-320, 2013.

18. Ujiki MB, Nayar R, Sturgeon C and Angelos P: Parathyroid cyst: Often mistaken for a thyroid cyst. World J Surg 31: 60-64, 2007.

19. Shields TW and Immerman SC: Mediastinal parathyroid cysts revisited. Ann Thorac Surg 67: 581-590, 1999. 